\title{
Acquisitions, traitements et analyses de données de courants, vagues et hauteur d'eau, pour l'amélioration de la gestion du risque de submersion marine : cas du PAPI Saint-Malo
}

\section{Léo SEYFRIED ${ }^{1}$, Héloïse MICHAUd ${ }^{1}$, Audrey PASQUET ${ }^{1}$, Fabien LECKLER ${ }^{1}$, Laurie BISCARA ${ }^{2}$, Christophe VRIGNAUD ${ }^{2}$}

1. Shom, Antenne de Toulouse, BP 45017 - 31032 Toulouse, France.

leo.seyfried@shom.fr

2. Shom, 13 rue du Chatellier, 29200 Brest, France.

\section{Résumé :}

À l'initiative de Saint-Malo Agglomération (SMA), un Programme d'Actions pour la Prévention des Inondations (PAPI) d'intention a été mis en place. Son axe 2 s'intéresse à l'amélioration de la connaissance des phénomènes météo-marins (vagues, houles, surcotes) au large de Saint-Malo. Pour y répondre, l'approche proposée est d'utiliser les moyens complémentaires que sont le recueil et l'utilisation de données in-situ d'une part, et la modélisation d'autre part. Dans un premier temps, une campagne de mesure océanographique d'envergure a été réalisée par le Shom durant l'hiver 2018-2019, en parallèle d'une campagne bathymétrique. Des données de courants, de vagues et de hauteur d'eau, ont été acquises à l'aide d'un réseau de 22 mouillages, pour un total de 37 instruments, dont 18 capteurs de pression, 7 profileurs de courant, 4 profileurs de courant houlographe, 4 courantomètres ponctuels, 2 marégraphes et 2 bouées houlographiques. L'ensemble du jeu de données acquis dans la baie de Saint-Malo, et leurs traitements sont présentés. Une première analyse des processus physiques observés est exposée. Les données acquises et les traitements effectués seront diffusés en open data sur le portail du Shom.

Mots-clés : Campagne de mesure, Hydrodynamique côtière, Traitements de données de pression, PAPI, Submersion marine.

\section{Introduction}

Le littoral malouin est particulièrement sensible aux aléas de submersion marine : 40 épisodes de submersion ont été identifiés entre 1703 et 2010 (PPRSM de Saint-Malo). Pour améliorer la connaissance de ces phénomènes et prévoir leurs impacts, la communauté de communes de Saint-Malo (SMA) a initié un Programme d'Actions pour la Prévention des Inondations (PAPI) d'intention. Pour répondre au besoin d'amélioration de la connaissance, la collectivité a pris contact avec le Shom en tant qu'expert national en appui aux politiques publiques de la mer et du littoral. L'approche proposée est d'utiliser les moyens complémentaires que sont l'observation et le recueil de données insitu d'une part, et la mise en place et l'utilisation de la modélisation d'autre part. 


\section{Thème 1 - Hydrodynamique côtière}

Cette première étude décrit la campagne de mesure mise en place par le Shom, pour améliorer la connaissance de l'hydrodynamique et des vagues du large jusqu'à la côte. L'ensemble du jeu de données recueilli a été traité avec différentes méthodes qui représentent l'état de l'art en termes de traitements de données océanographiques, notamment en ce qui concerne le traitement des données de pression de fond. Une première analyse de ce jeu de données est présentée à travers l'analyse d'un événement tempétueux et d'un événement de grande marée. Les données acquises et les traitements effectués seront diffusés en open data sur le portail du Shom.

\section{Présentations des données acquises}

\subsection{Site d'étude}

Le site d'étude (figure 1) est une baie peu profonde, relativement complexe car sujette à un régime de marée méga-tidal (marnage supérieur à $12 \mathrm{~m}$ ) et des conditions estuariennes modifiées par la présence d'une usine marémotrice. Les courants de marée sont orientés E-SE au flot et O-NO au jusant. Les vagues se propagent principalement dans la zone ONW et N-NW, avec des hauteurs pouvant atteindre $7 \mathrm{~m}$ au large lors des tempêtes hivernales (PPRSM de Saint-Malo). Les interactions avec les nombreux obstacles (îlots, hauts fonds, épis) sont encore peu connues et limitent encore la compréhension de l'hydrodynamique dans la baie (DUCATEZ et al., 2018).

\subsection{Instrumentation}

La baie a été instrumentée par le Shom de façon dense et durable pendant l'hiver 20182019 à l'aide 22 mouillages (figure 1), pour un total de 37 instruments. Deux bouées de mesure de houle directionnelles (Datawell, DWR-MkIII) ont été positionnées au large, pour évaluer le champ de vagues entrant dans la baie. Pour compléter les informations de hauteur d'eau fournies par le marégraphe officiel du port de Saint-Malo (réseau de marégraphe RONIM), deux marégraphes à pression (Seabird Electronics, SBE26+) ont été positionnés à une profondeur de $10 \mathrm{~m}$ (Cézembre et La Plate). En complément, 18 capteurs de pression haute fréquence (Ocean Sensor System Inc., 10 OSSI-010-003C et 8 OSSI-010-022) ont été installés le long de quatre segments (T1, T2, T3 et T4 sur la figure 1). Ces capteurs nous informent sur les hauteurs d'eau et les vagues dans la baie peu profonde, sur l'estran et jusqu'au pied de la digue (de $-12 \mathrm{~m}$. à $5 \mathrm{~m}$, en convention IGN69). Les capteurs de pression sont combinés avec des profileurs de courant (Nortek, Aquadopp profiler $1 \mathrm{MHz}$, AquaPro) dans la baie peu profonde et des courantomètres ponctuels (Nortek, Aquadopp single-point current meter, AquaDopp) sur l'estran. Quatre profileurs de courant houlographe (Nortek, AWAC $600 \mathrm{kHz}$ ) ont été également déployés, avec les marégraphes et des OSSI, à des profondeurs de l'ordre de $12 \mathrm{~m}$. 


\section{XVİ̀mes Journées Nationales Génie Côtier - Génie Civil \\ Le Havre 2020}

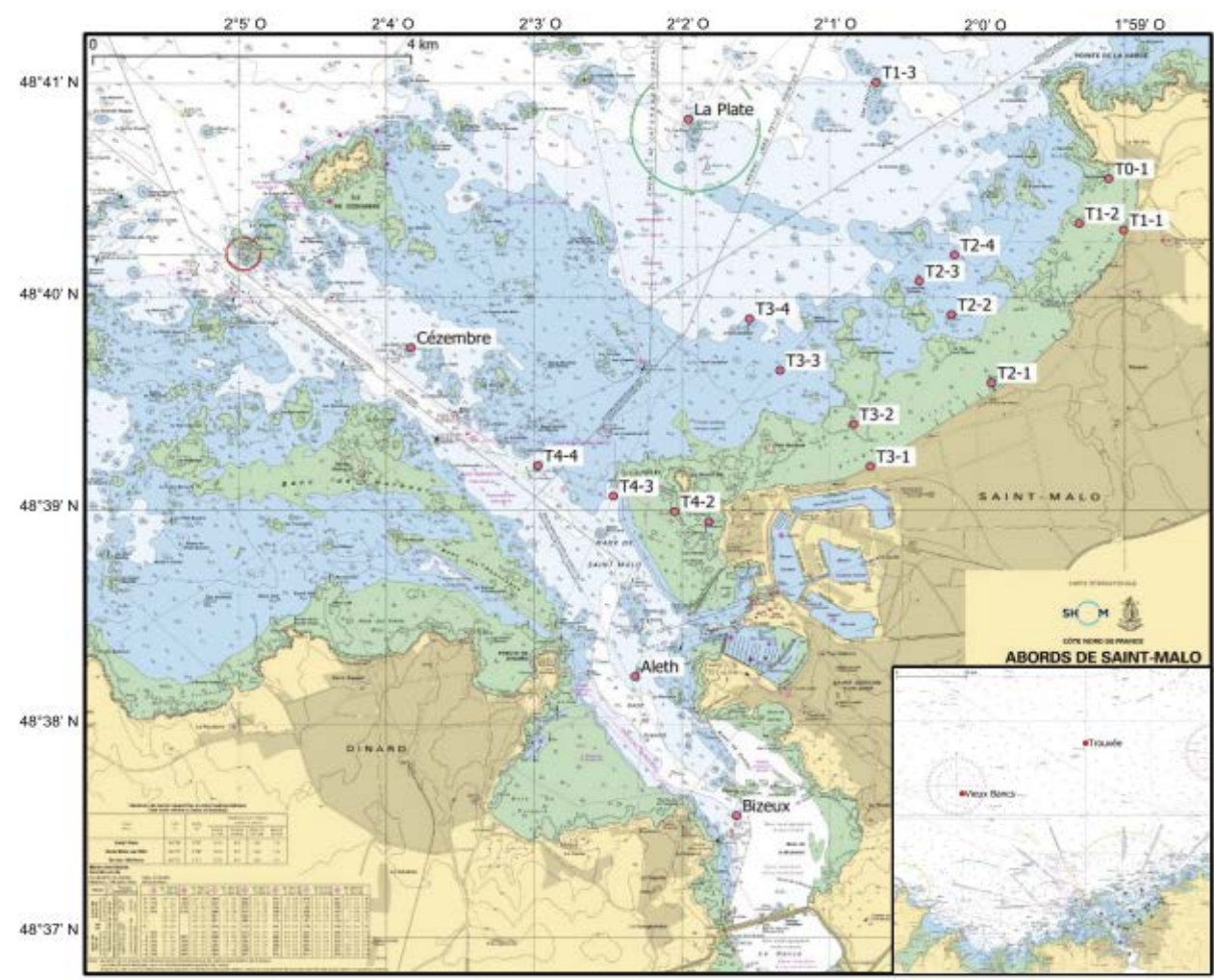

Figure 1. Carte des mouillages déployés dans la baie de Saint-Malo.

\subsection{Bilan d'acquisition des instruments}

L'acquisition des données s'étale sur la période entre le novembre 2018 et avril 2019. La figure 2 présente un bilan d'acquisition des différents instruments pour chacun des mouillages. On remarque des interruptions dans l'acquisition des OSSI des segments T2 et $\mathrm{T} 3$ dues à des batteries moins performantes que prévues initialement sur les OSSI de nouvelle génération (OSSI-010-022). Un changement de batterie a été effectué du 22/01/2019 au 24/01/2019 pour les capteurs positionnés sur l'estran uniquement. L'AWAC du mouillage La Plate n'a acquis aucune donnée suite à une défaillance du câble d'alimentation de l'instrument. Le mouillage T3-4 a quant à lui disparu en cours de campagne, aucune donnée n'a pu être récupérée. Malgré ces incidents (faisant partie des aléas des campagnes océanographiques), la densité et la complémentarité des instruments mis en œuvre nous permettent d'avoir une description de l'hydrodynamique et de l'état de mer dans l'ensemble de la baie de Saint-Malo pendant une période hivernale de plus de 4 mois. 


\section{Thème 1 - Hydrodynamique côtière}

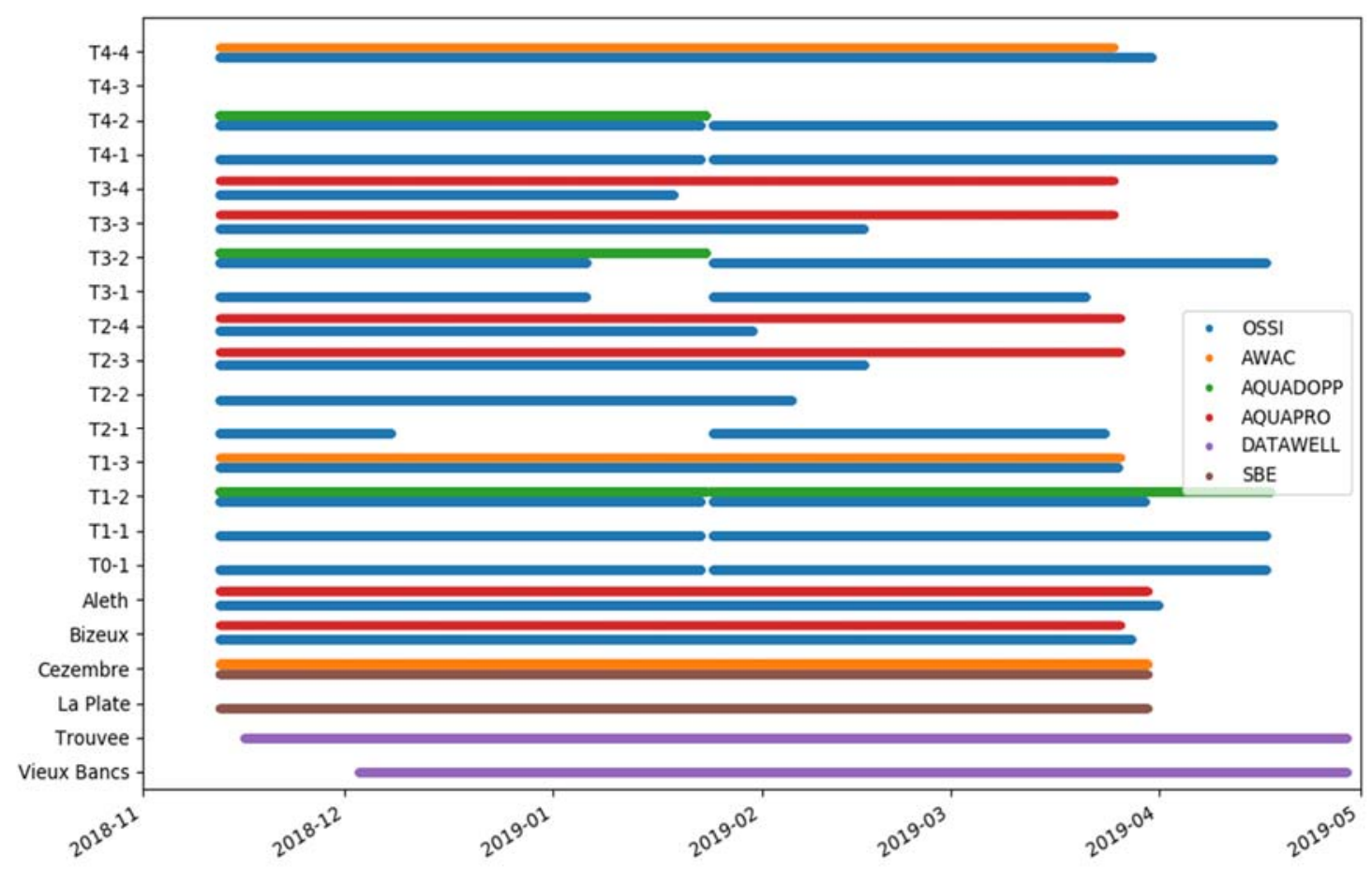

Figure 2. Bilan d'acquisition des instruments.

\section{Traitements des données}

\subsection{Données de hauteur d'eau}

Dans cette étude, les hauteurs d'eau sont reconstruites à partir des mesures de pression des marégraphes SBE, des capteurs haute fréquence OSSI et des courantomètres Nortek. Pour reconstruire la hauteur d'eau depuis les mesures de pression, chaque capteur est étalonné en comparant la pression mesurée à l'air libre avec la pression atmosphérique au niveau de la mer (figure 3.a). Le signal de pression atmosphérique est ensuite retranché au signal de pression précédemment étalonnée. La hauteur d'eau est finalement déduite par approximation hydrostatique (figure 3.b). Pour les capteurs de pression haute fréquence, la hauteur d'eau est lissée, avec une moyenne glissante de $10 \mathrm{mn}$, afin de filtrer les déformations de la surface de l'eau liées aux vagues (figure 3.b).

\subsection{Données de courants}

Dans cette étude, les courants sont mesurés en utilisant trois types de courantomètres à effet Doppler Nortek (AquaDopp, AquaPro et AWAC) dont les données sont traitées de façon similaire par le logiciel constructeur Storm. Ils sont reprojetés sur les axes nordest-vertical et les valeurs mesurées hors de l'eau sont supprimées en utilisant la mesure de hauteur d'eau du capteur de pression (figure 3.c). Les mesures proches de la surface et polluées par les lobs secondaires des faisceaux acoustiques sont supprimées. Avant leurs 


\section{XVI'̀mes Journées Nationales Génie Côtier - Génie Civil \\ Le Havre 2020}

déploiements, les compas magnétiques de ces courantomètres sont préalablement étalonnés sur une plateforme dédiée du Shom.

\subsection{Données de vagues}

Dans cette étude, les données de vagues sont mesurées et reconstruites de façons différentes selon le type d'instruments (Datawell, OSSI et AWAC).

Les Datawell sont directement traitées à l'aide du logiciel constructeur Wave21. Les données de déplacements verticaux et horizontaux sont filtrées et traitées pour en déduire les spectres directionnels des vagues.

Les OSSI permettent de reconstruire l'élévation de la surface libre à partir du signal de pression haute fréquence. MOURAGUES et al. (2019) proposent une revue des différentes méthodes de reconstructions non hydrostatiques. La méthode la plus utilisée est la méthode linéaire basée sur le facteur de correction non-hydrostatique $K_{p}$ (Equation 1). Une limitation importante de cette méthode est l'introduction d'une fréquence de coupure (de l'ordre de $0.3 \mathrm{~Hz}$ ) pour limiter la divergence de la méthode à haute fréquence induite par la relation de dispersion (Equation 2).

$F\left\{\zeta_{l}\right\}=K_{p}(\omega) F\left\{\zeta_{h}\right\} \operatorname{avec} K_{p}(\omega)=\frac{\cosh \left(k(\omega) h_{0}\right)}{\cosh \left(k(\omega) \delta_{m}\right)}$

où : $F\{$.$\} la transformée de Fourier, \zeta_{h}$ l'élévation de la surface libre par approximation hydrostatique, $\omega$ la pulsation, $\mathrm{k}$ le nombre d'onde, $\delta_{m}$ la profondeur du capteur et $h_{0}$ la profondeur moyenne. $\omega$ et $\mathrm{k}$ sont reliés par la relation de dispersion suivante :

$\omega^{2}=\operatorname{gktanh}\left(k h_{0}\right)$

En eau peu profonde, les vagues deviennent fortement non-linéaires et faiblement dispersives. L'utilisation de la méthode linéaire est discutable. BONNETON et al. (2018) proposent une nouvelle méthode de reconstruction non linéaire faiblement dispersive de l'élévation de la surface libre (Equation 3).

$\zeta_{s w n l}=\zeta_{s w l}-\frac{1}{g}$

où : $\zeta_{s w l}$ l'élévation de la surface libre par méthode linéaire faiblement dispersive :

$\zeta_{s w l}=\zeta_{h}-\frac{h_{0}}{2 g}\left(1-\left(\frac{\delta_{m}}{h_{0}}\right)^{2}\right) \partial_{t}^{2} \zeta_{h}$

Cette méthode permet une meilleure reconstruction de la hauteur des plus grandes vagues (MOURAGUES et al., 2019), cependant son application est limitée à un régime de vagues faiblement dispersif $\left(\mu=\left(k h_{0}\right)^{2}<0.3\right)$. L'élévation de surface reconstruite avec les différentes méthodes (figure 3.d) est ensuite traitée par analyse spectrale et analyse vagues à vagues pour en déduire les paramètres des vagues (figure 3.e).

Les profileurs de courant houlographe (AWAC) permettent de reconstruire un spectre de vagues directionnel à partir des mesures de courants orbitaux et de la pression par méthode PUV. Pour traiter ces données, on utilise le logiciel constructeur Quickwave dans lequel la méthode précédente est implémentée. 


\section{Thème 1 - Hydrodynamique côtière}
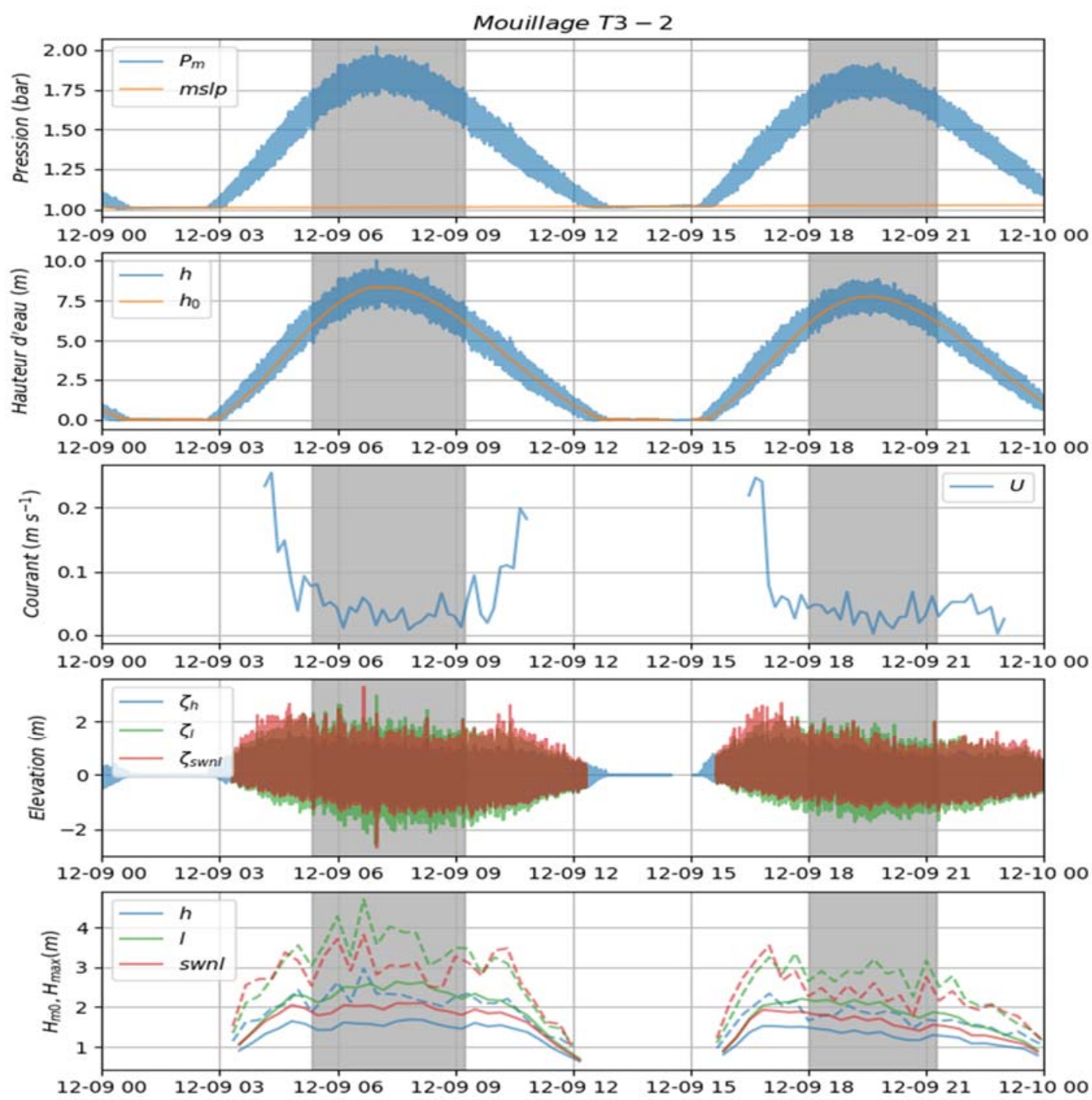

Figure 3. Série temporelle des données du mouillage T3-1 pendant l'événement tempétueux du 9 décembre 2018. (a) pression haute fréquence mesurée $\left(P_{m}\right)$ et pression atmosphérique (mslp), (b) hauteur d'eau, au-dessus du capteur, reconstruite par approximation hydrostatique $(h)$ et lissée $\left(h_{0}\right)$ à partir de $P_{m},(c)$ intensité du courant mesuré, (d) élévation de la surface libre, reconstruite à partir de $P_{m}$ par méthode hydrostatique $\left(\zeta_{h}\right)$, linéaire $\left(\zeta_{l}\right)$,.et faiblement dispersive non linéaire $\left(\zeta_{\text {swnl }}\right)$, (e) hauteur significative $\left(H_{m 0}\right.$, en trait plein) et hauteur maximum des vagues $\left(H_{\text {max }}\right.$, en pointillé) calculées respectivement par analyse spectrale et vague à vague, pour les reconstructions hydrostatique $(h)$, linéaire (l), et faiblement dispersive non linéaire (swnl). Les bandes grises correspondent à des périodes où $h 0>6 \mathrm{~m}$.

\section{Résultats}

Au cours de l'événement tempétueux du 9 décembre 2018, deux cycles de marée sont observés avec une hauteur d'eau au-dessus du capteur qui varie de 0 à $8 \mathrm{~m}$ (figure 3.b). 


\section{XVI èmes Journées Nationales Génie Côtier - Génie Civil \\ Le Havre 2020}

Au début et à la fin des cycles de marée $\left(h_{0}<6 \mathrm{~m}\right)$, on peut voir que l'élévation de la surface (figure 3.d) et la hauteur maximum des vagues (figure 3.e) les plus importantes sont reconstruites par la méthode non-linéaire faiblement dispersive (en rouge). Au centre des cycles de marée, les valeurs les plus importantes sont reconstruites par la méthode linéaire (en vert). À cette profondeur $\left(h_{0}>6 m\right)$, la validité des méthodes faiblement dispersives est discutable. En ce qui concerne la hauteur significative, les différences entre les méthodes sont faibles au début et à la fin des cycles de marée et plus importantes à profondeur intermédiaire avec des hauteurs significatives maximum pour la méthode linéaire. Pour l'OSSI du mouillage T3-1 (non montré), qui se trouve à une hauteur d'eau qui varie entre 0 et $2 \mathrm{~m}$, les hauteurs maximales déduites de la méthode non linéaire peuvent être supérieures de $30 \%$ à celles déduites de la méthode linéaire, bien que les hauteurs significatives soient faiblement impactées. Dans la suite de cette étude, nous utiliserons uniquement la méthode linéaire, bien qu'elle sous-estime probablement la hauteur maximum des vagues proche de la côte.

Les données traitées nous permettent d'avoir une vision de la hauteur d'eau et du champ de vagues dans la baie de Saint-Malo (figure 4). Dans cette étude, nous nous intéressons à deux événements qui se sont produits durant la campagne de mesure. Le premier événement est la tempête du 9 décembre 2018 discuté précédemment, le second événement est un épisode de grande marée survenu le 22 février 2019 (coefficient de marée 113). Le 9 décembre, le 99ème quantile de la hauteur d'eau observé à la côte varie entre un minimum de 5,4 $\mathrm{m}$ au capteur T4-1 et un maximum de 5,7 $\mathrm{m}$ à Rocabey (capteur T3-1). Ces hauteurs d'eau correspondent à un faible coefficient de marée auquel s'ajoute une surcote atmosphérique qui peut atteindre $60 \mathrm{~cm}$ au marégraphe du port de Saint-Malo. Pendant cet événement, le champ de vagues est développé dans toute la baie, avec des hauteurs significatives de plus de $4 \mathrm{~m}$ au large (capteur T1-3), et des hauteurs de plus de $1,7 \mathrm{~m}$ à la côte (capteurs T4-1 et T1-1). Le 22 février, le 99ème quantile de la hauteur d'eau observé atteint un maximum de $6.9 \mathrm{~m}$ à Rocabey. Le champ de vagues est faiblement développé avec des hauteurs significatives inférieures à $1,2 \mathrm{~m}$ au large (capteur T1-3), mais à la côte, on observe quand même des hauteurs de plus de 1,3 m (capteurs T1-1, T2-1 et T3-1). Des franchissements par paquet de mer ont été observés à Rochebonne. 


\section{Thème 1 - Hydrodynamique côtière}
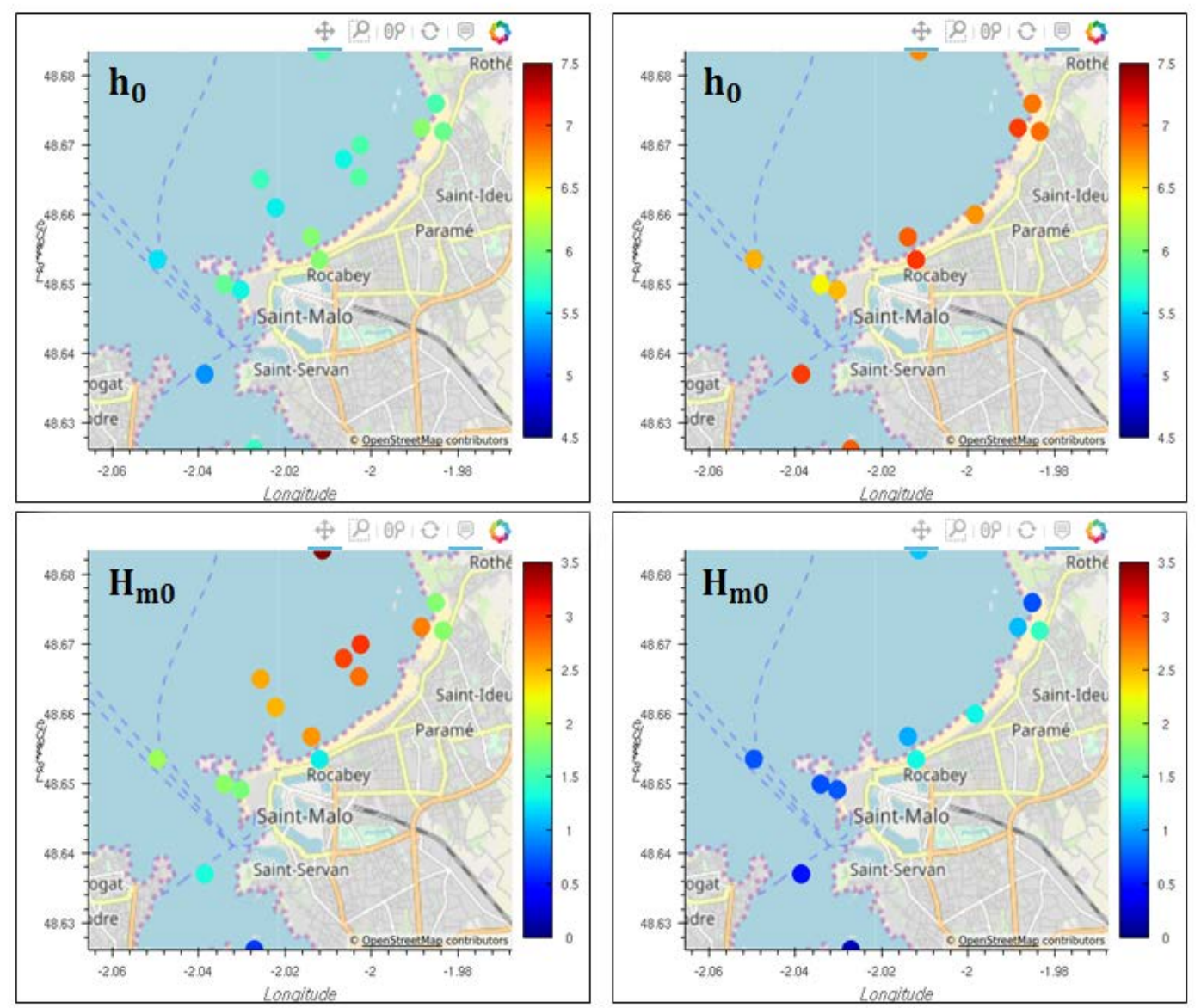

Figure 4. Cartes du 99ème quantile de la hauteur d'eau $h_{0}$ (en haut) et de la hauteur significative des vagues $H_{m 0}$ reconstruites par méthode linéaire (en bas), déduites des mesures de pression des OSSI, pendant l'épisode tempétueux du 9 décembre 2018 (à gauche) et pendant l'épisode de grande marée du 22 février 2019 (à droite).

\section{Conclusions}

Dans une baie sujette à un régime méga-tidal, le choix de la méthode de reconstruction de l'élévation de la surface libre à partir de la pression est fortement dépendant de la phase de la marée. En absence de mesure directe de l'élévation, à partir des résultats actuels, il est difficile de conclure sur la méthode à appliquer. Actuellement, la meilleure solution repose sur la combinaison des différentes méthodes, en faisant attention à la transition entre les méthodes. Pour les capteurs évoluant à faible profondeur, les résultats montrent des écarts entre les méthodes semblables à ceux mesurés par MOURAGUES et al. (2019). L'utilisation de la méthode non-linéaire faiblement dispersive est à privilégier pour étudier l'impact des vagues de submersion sur la digue. Pour les profondeurs intermédiaires, la méthode linéaire est à favoriser.

L'analyse de deux événements illustre les conditions favorisant le risque de submersion, surcotes atmosphériques et fortes vagues couplées à une grande marée. Durant la 


\section{XVIèmes Journées Nationales Génie Côtier - Génie Civil \\ Le Havre 2020}

campagne, aucun événement tempétueux n'est intervenu durant un événement de grande marée. Dans la suite du projet, la modélisation numérique permettra d'étudier le phasage et les interactions entre ces événements.

Cette campagne de mesure océanographique, combinée à la bathymétrie haute résolution acquise parallèlement (BISCARA et al., 2020), fournit un ensemble de données cohérent et uniques pour la validation des modèles et les études de processus dans une baie sujette à une marée méga-tidale. Dans le cadre du projet PAPI Saint-Malo, ces données permettront la mise en place et la validation d'un modèle couplé vague-surcote à fine échelle (résolution cible autour de $30 \mathrm{~m}$ ) en cours de développement au Shom.

\section{Remerciements}

Cette étude a été financée par le Shom, SMA, le Conseil départemental d'Ille et Vilaine (CD35) et l'Etat français. Les auteurs remercient les équipes du SHOM/GHOA, $S H O M / D M G S / I E S$ et toutes les personnes ayant participé à la campagne de mesure.

\section{Références bibliographiques}

BONNETON P., LANNES D., MARTINS K., MICHALLET H. (2018). A nonlinear weakly dispersive method for recovering the elevation of irrotational surface waves from pressure measurements. Coastal Engineering, Vol. 138, pp 1-8, https://doi.org/10.1016/j.coastaleng.2018.04.005

BISCARA L., SALVATERRA C., ROSETTO J., LE JEUNE S., MICHAUD H., SEYFRIED L., PASQUET A., VRIGNAUD C. (2020). Production de MNT topobathymétriques pour l'amélioration de la gestion du risque de submersion marine : cas du PAPI Saint-Malo XVI ${ }^{\text {ème }}$ Journées Nationales Génie Côtier-Génie Civil, 9-11 juin 2020, Le Havre, 8 p., sous presse.

DUCATEZ J. P., LECORNEC E., KERAMBRUN G., VIDAL S. (2018). Coastal risk management plan in Saint-Malo. In: Gourbesville P., Cunge J., Caignaert G. (eds), Advances in Hydroinformatics, Springer Water, Springer, Singapore, pp 621-627, https://doi.org/10.1007/978-981-10-7218-5_44

MOURAGUES A., BONNETON P., LANNES D., CASTELLE B., MARIEU V. (2019). Field data-based evaluation of methods for recovering surface wave elevation from pressure measurements. Coastal Engineering, Vol. 150, pp 147-159. https://doi.org/10.1016/j.coastaleng.2019.04.006 
Thème 1 - Hydrodynamique côtière 